பாவை இலக்கியங்களின் இறைநெறிக் கொள்கைகளும், கோட்பாடுகளும்

சே. சித்ரா அ, •

அ தமிழ்த்துறை, கே.ஆர்.கலை மற்றும் அறிவியல் கல்லூரி, கே. ஆர். நகர், கோவில்பட்டி-628503, தமிழ்நாடு, இந்தியா

\title{
The Theological Teachings and Principles of Pavai Literature
}

\author{
S. Chitra ${ }^{a}$ * \\ ${ }^{a}$ Department of Tamil, KR College of Arts \& Science, K.R.Nagar, Kovilpatti-628503, Tamil Nadu, India
}

* Corresponding Author: chithiram62@gmail.com

Received: 04-01-2020 Revised: 17-04-2021

Accepted: 19-04-2021

Published: 30-04-2021

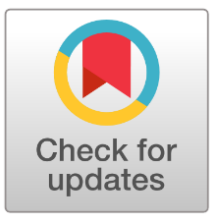

\section{ABSTRACT}

The article analyzes the turn of the year in recognition of Kannaberumaan, the Nandavegopan Kumaran, the sharpen sward tycoon, and Yasodai, the young lion, and the Thiruvemba of Manivasaka, who was dissolved by the Thiruvasaka that I am a liar, my heart is a lie, and i cry. Both the creators of reverential literature have recorded the rise of the female race through songs of early morning, expressing the principles of the standards and unmistakable rules that blasphemy is raised despite the fact that religious thoughts are at various levels.

Keywords: Pavai Literature, Andal, Perumal, Yasodai

\section{முன்னுரை}

மொழி மனிதனின் எண்ணங்களை எடுத்துக்காட்டுவதோடு அமையாது, அது அவனின் மனவளத்தையும் வடித்துக்காட்டும் கருவியாகின்றது. பேச்சில் கருத்துக்களைப் பரிமாறிக் கொள்ளப் பயன்பட்ட மொழி, எழுத்து இலக்கியமாக்கப்பட்ட பொழுது, அது பிற்காலச் சமுதாயத்தாருக்கு வழிகாட்டியாகவும் மாறியது. அந்நிலையில் எழுந்த தமிழ்மொழி இலக்கியங்களில் காதல், வீரம், அறம், பக்தி, புதுமை எனப் பல்வகை பாடுபொருள் வடிவங்களை எடுப்பினும், குறிப்பாக 'பக்தியின் மொழி தமிழ் மொழி எனத் தனிச்சிறப்புடன் பெயர் பெற்று இலங்குகின்றது. அவ்வழியில் தொன்மை இலக்கியமான தொல்காப்பியம் அகப்பொருளில் நிலவரையறை அளிக்கும் முறையினிலே கடவுளரை இணைத்துச் சொல்லும் முறைமை வெளிப்பட்டுள்ளது காணலாம்.

"மாயோன் மேய காடுறை உலகமும்

சேயோன் மேய மைவரை உலகமும்

வேந்தன் மேய தீம்புனல் உலகமும்

வருணன் மேய பெருமணல் உலகமும்

சொல்லிய முறையால் சொல்லவும் படுமே"

என்று நால்வகை நிலவழிபாட்டுக்குரிய இறையை இணைத்துக் காட்டியது (llampuranar, 1982). அவ்வகையில் தொல்காப்பியந் தொட்டு இன்றுள்ள இலக்கியங்கள் வரை உள்ள படைப்பாளர்கள் தமிழ் 
மொழியையும், இறையையும் தனித்தனியே பிரிக்க இயலாது என்பதை உணர்த்தியுள்ளனர். அந்நெறியினில் எழுந்த பக்தி இலக்கியங்களான ஆண்டாளின் திருப்பாவையும், மணிவாசகப் பெருமானின் திருவெம்பாவையும் பக்திநெறியில் பெண்களைச் செலுத்தியதோடு, மார்கழித் திங்களை அப்பெண்மைக்கு ஏற்றம் தரும் திங்களாக்கியுள்ளன.

\section{பாவை இலக்கியங்களின் கொள்கைகள்}

பரம்பொருள் ஒன்றே. அவனோடு மண்ணில் வாழும் பொழுதும், அதன் பின்னும்; இணைந்தே இருக்க வேண்டும் என்பது இவ்விலக்கியங்களின் அடிக் கொள்கைகளாகின்றது. கொண்ட கொள்கைக்காக பாவையர் செயல்பட்ட செயல்பாடுகளே, பற்றிய, உறுதியான இறைக்கோட்பாடுகளாகின்றன. அவர்களின் வினைகளில் மார்கழிநோன்பு எனும் வைகறைநோன்பும், அதற்கெனத் துயில் எழல், நீராடல், பரம்பொருள் பற்றி பேசல், போற்றல், பின் அவனுக்கே உற்றவராதல், சமுதாயப் பயன் கருதி வேண்டல், இயற்கையை இறைவனாக்குதல் எனப் பல மேன்மை தரும் கிரிசைகள் (கடமைகள்) அதனுள் வரையறுக்கப்பட்டுள்ளன. அவ்வகையில் அப்பாவையர் கொண்ட காலத் தெளிவு, கடமைகள், வேண்டல் என்ற நிலைகளின் கீழ் இக்கட்டுரை காணப்பெற்று உள்ளது.

\section{காலத்தெளிவு}

தற்காலத்தில் ‘காலமே காசு’ எனவும், ‘காலமே கடவுள்’ என்பதும்; பொருளாதாரத்தில் முன்னேறிய ஒவ்வொரு நாட்டின் மொழிகளாகின்றது. காலத்தின் அருமையை அளந்து சொன்ன வள்ளுவரும்,

“ஞாலம் கருதினும் கைகூடும் காலம்

கருதி இடத்தாற் செயின்” என்றும்,

அருவினை என்ப உளவோ கருவியான்

காலம் அறிந்து செயின்" என்றும்

தெளிவுபடுத்தினார் (Ilankumaranar, 2007). அவ்வழி வந்த ஒளவையும், 'பருவத்தே பயிர் செய்' என்று வலியுறுத்தியுள்ளார் (Avvaiyar, Bharathiar, Bharathidasan, 2018).

இறை வணக்கத்துக்கு வைகறை ஏற்ற பொழுதாகும். அதிலும் மார்கழியின் வைகறை தூய்மையும், உயர்வு பெற்றதாகும். எனவேதான் ஆண்டாள் நாச்சியார்,

“மார்கழித் திங்கள் மதிநிறைந்த நன்னாளால்

நீராடப் போதுவீர் போதுமினோ நேரிழையீர்"

என்று நீராட்டத்தின் முதல் அழைப்பை விடுக்கின்றார் (Andal, 2010). அறிவியல் வழி ஓசோன் படலம் நன்மைப் பயக்கும் வகையில் நிலைபெற்ற திங்கள் மார்கழியாம். அதனாலேயே அத்திங்களில் செய்யப் பெறும் வைகறை நோன்பு மிக ஏற்றது எனும் வகையில் அழைப்பு தரப்பட்டுள்ளது. நற்செயலைச் செய்யத் தொடங்கும் வேளையில் யாருக்கும் அதிகாலை உறக்கம் பெருந்தடையாக அமையும். அதனால் ஆண்டாள் அதனை நீக்குக என்றாள். அதனைப் பின்வருமாறு பாடலில் பொருளமைத்து பாடியுள்ளார்.

“தூமணி மாடத்துச் சுற்றும் விளக்கெரியத்

தூபம் கமழத் துயிலணைமேல் கண்வளரும்

மாமான் மகளே! மணிக்கதவம் தாள் திறவாய்

மாமீரவளை யெழுப்பீரோ? உன் மகள் தான் 
ஊமையோ அன்றிச் செவிடோ அனந்தலோ

ஏமப்பெருந்துயில் மந்திரப்பட்டாளோ"

என்று துயிலை மனித வளத்திற்கு இறுதி கூறும் நிலை என்கிறாள். இங்கு உறக்கத்திற்கு வாய்ப்பானச் சூழல்கள் கூறப்பட்டுள்ளன (Andal, 2010). அதனுள் மூழ்கினால் இறுதி உறக்கம் எனும் 'ஏமப் பெருந்துயில்' நிலைக்குத் தள்ளப்படுவீர் என்ற முன் அறிவிப்புச் சொல் விடுக்கின்றாள் ஆண்டாள். மேலும் மிகத்துயில் கொள்வோரை கும்பகரணனாாடு ஒப்பிடுதல் வழக்கம். ஆதலால்,

“கூற்றத்தின்வாய் வீழ்ந்த கும்பகரணனும்

தோற்றுமுனக்கே பெருந்துயில் தந்தானோ"

என்று துயில்மேல் ஈடுபாடுடைய பெண்ணை எழுப்புகின்றாள் (Andal, 2010). இதனால் ஆற்றலும், வலிமையும், வரமும் மிக்க கும்பகர்ணனும் துயிலால் அழிந்தான். அதுபோல் நீயும் அழிய வேண்டாமென்ற நல்லுணர்வில் எழுப்புகின்றது பாவைப் பாடல் என்பது புலனாகின்றது.

“திருவெம்பாவையும் ஆதியும் அந்தமும் இல்லாத

அரும்பெருஞ் சோதியைப் பாடக் கேட்டும்,

மாதே வளருதியோ வன்செவியோ நின்செவிதான்"

என்று உறக்கத்தால் செல்வத்துள் செல்வமாகிய செவிச்செல்வம் இழந்து போனவளாக்கி, பின்பிறவிப்பயனும் இழந்துபோனதை மணிவாசகர் வெளிப்படுத்துகின்றார். மேலும் உறங்கினால் வாழும்காலம் வீணாகும் என்பதையும் பின்வரும் நிலையில் உணர்த்துகின்றார் (Andal, 2010).

“கண்ணைத் துயின்றவமே காலத்தைப் போக்காதே”

என்கிறது பாவைப்பாடல். எனவே இறைவணக்கத்திற்கு உரிய முதல் தடை உறக்கம் எனச் சொல்லி அதனை விடுக்க என்கின்றன பாவைப் பாடல்கள் (Andal, 2010).

\section{கடமைகள்}

‘ஈன்று புறந்துரும்’ தாய்மைக்குணம் கொண்ட பெண் இனத்தின் கடமையை, பணிவிடைகளை நோன்பு நோற்கும் வழி பின்பற்றுதல் வேண்டும். அது பெண்மைக்கு ஏற்றமும், பழி போக்கி நற்பண்புகளை வளர்க்கும். உயர்வான பண்புகளைப் பெற்ற பாவையே இறைவர்க்கு பணி செய்ய இயலும். அதனால் பின்வரும் கிரிசைகள் கேளீரோ என்று பெண்ணினத்திற்கு அழைப்பு விடுக்கின்றாள் பூங்கோதையார்.

“வையத்து வாழ்வீரீகாள்! நாமும் நம் பாவைக்குச்

செய்யும் கிரிசைகள் கேளீரோ, பாற்கடலுள்

பையத்துயின்ற பரமனடி பாடி

நெய்யுண்ணோம் பாலுண்ணோம் நாட்காலே நீராடி

மையிட்டெழுதோம் மலரிட்டு நாம் முடியோம்

செய்யாதன செய்யோம் தீக்குறளை சென்றோதோம்

ஐயமும் பிச்சையும் ஆந்தனையும் கைகாட்டி

உய்யுமாறெண்ணி உகந்தேலோர் எம்பாவாய்"

என்ற பாசுரத்தால் பெண்கள் வையத்தில் நிலைபெற்று வாழக் கடமைகள் என்னவெலாம் செய்தல் வேண்டும் என்று பட்டியல் இட்டுச் சொல்வதனைக் காண முடிகின்றது. இறை வணக்கத்தைச் செலுத்துபவர்கள் வளமானப் பொருள்களைத் தவிர்த்து, எளிமையுடன் திகழ்ந்து, செய்யக் கூடாதவற்றை 
விடுத்து ஏழை, எளியவர்க்கு உணவிட்டு உய்யும் வழிக் காணுதல் வேண்டுமென்கின்றது பாடல். திருவெம்பாவையும், “உன்னடியார் தாள் பணிவோம்” என்கிறது (Andal, 2010).

\section{வேண்டல்கள்}

பொதுநலமாம் சமூக நலன் கருதிய வேண்டல்களாக இறைவணக்கம் அமைந்துள்ளதைக் காண முடிகின்றது. (ஆண்டாளின்) ஓங்கியுலகளந்த உத்தமன் தன் புகழ் பாடினால், நாட்டில் பசுமை வளம், நீர்வளம், பால்வளம், அமைதி ஆகியவை நிலை பெறும் என்கிறது பாசுரப்பாடல்.

“தீங்கின்றி நாடெல்லாம் திங்கள் மும்மாரி பெய்து

ஓங்கு பெருஞ்செந்நெல் ஊடு கயலுகள

பூங்குவளைப் போதில் பொறிவண்டு கண்படுப்பத்

தேங்காதே புக்கிருந்து சீர்த்தமுலை பற்றி

வாங்கக் குடம்நிறைக்கும் வள்ளற் பெரும்பசுக்கள்

நீங்காத செல்வம் நிறைந்தேலோர் எம்பாவாய்!"

என்று பாவை நோன்பு புரிய ஆர்வம் ஊட்டுகின்றது (Andal, 2010).

மணிவாசகப் பெருந்தகையின் வேண்டல்கள், மண் உலகத்தை கடந்தது. ‘என் கடன் பணி செய்து கிடப்பதுவே' என நாவுக்கரசர் பெருமான் கூறுவது ஒத்தது.

“இப்பிறப்பறுத்து எமை ஆண்டருள் புரியும் எம்பெருமான்"

என்று பள்ளியெழுச்சி பாடும் பாவையர்கள்,

“உங்கையிற் பிள்ளை உனக்கே அடைக்கலமென்று

அங்கப் பழஞ்சொல் புதுக்கும்எம் அச்சத்தால்

எங்கள் பெருமான் உனக்கொன் றுரைப்போம் கேள்

எம் கொங்கை நின்னன்ப ரல்லார் தோள் சேரற்க

எங்கை உனக்கல்லா தெப்பணியும் செய்யற்க

கங்குல் பகலெங்கண் மற்றொன்றும் காணற்க

இங்கிப் பரிசே எமக்கெங்கோன் நல்குதியேல்

எங்கெழிலென் ஞாயி றெமக்கேலோ ரெம்பாவாய்"

என்று தன்னைச் சுற்றியே வேண்டல்களை அமைத்து உள்ளனர் (Andal, 2010). மேலும் இறையுடன் ஒன்ற, இல்லறத்திலும் துணைவன் சிவனன்பனாய் அமைந்ததால் அதுவே தனக்கு கிடைத்த பெரும் பேறாய் ஆகிவிடும் என்று எண்ணமிட்ட பெண் குரலாக உளது.

இயற்கையை இறைவனாய்க் காணும் நிலை இருவரின் பாடல்களிலும் நிலைத்துள்ளது. கோதை நாச்சியார் பாசுரத்தில், “ஆழி மழைக் கண்ணா ஒன்றும் நீ கைகரவேல்" 15 எனப்பாடி, மேகம் உருப்பெற கடலுள் நீர்முகந்து, திருமாலின் சங்கு, சக்கரம் போல் மின்னி, அதிர்ந்து, ஊழி முதல்வனாக உடல் கறுத்து அவனருள் போல் பார் வளம் பெற மழை பொழிய வேண்டும் என வேண்டப்படுகின்றது. திருவெம்பாவையின் மழையோ உமையம்மையின் அருள் வெள்ளமாகப் பொழிதல் வேண்டும் என்ற வேண்டுகோளாய்த் திகழ்வதைக் காணலாம். 


\section{முடிவுரை}

நாச்சியார் மொழி, மணிவாசகர் மொழி என்ற இரண்டும் பாவைப் பாடலைப் பாடினாலும் இரண்டிலும் ஒற்றுமையும் வேற்றுமையும் உண்டு என்பது மேல் அமைந்துள்ள சில பாடல்களால் உணரலாம். ஆனால் இரண்டுமே பரம்பொருள் அடைய உறுதி வேண்டும் என்பது வலியுறுத்தப்படுகின்றது. தமிழுக்கு ஏற்றம் தரும் 'ழ’ கரத்தை ஆண்டாள் மழைப்பாடலிலும், மணிவாசகர் வைகறை வரவிலும் பத்து இடங்களில் பயன்படுத்தி தாய்மொழிக்குப் பெருமையைச் சேர்த்துள்ளனர். பரம்பொருள் எனும் உறுதிப் பொருளை அடையும் கொள்கையில் இற்றைக்கும் ஏழ் ஏழ் பிறவிக்கும் உமக்கே உற்றோமே ஆவோம் என்ற உறுதிப்பாடு ஆண்டாளிடம் நிறைந்துள்ளது. மணிவாசகரிடம் இந்த பூமி சிவனுய்யக் கொள்கின்ற ஆறு என்று நோக்கி ஆட் கொள்ள வள்ளாய்" என இறைஞ்சும் நன்றியுணர்வைக் காண முடிகின்றது. திருப்பாவை முப்பதாம் பாடல் முடிவில் 'பட்டர்பிரான் கோதை சொன்ன சங்கத்தமிழ் மாலை முப்பதும்' என்று அடையாளமிடப்பட்டுள்ளது. பாமாலை சொன்னவர் செங்கண் திருமால் திரு அருள் பெற்று இன்புறுவர் என்று பாசுரப் பயனும் கூறப்பட்டுள்ளது. திருவெம்பாவையிலும் மூவர் அருளும், கருணையும் கிடைக்க, ஆட்கொள்ள திருப்பெருந்துறை ஆரமுதே பள்ளியெழுந்தருளாய் என்று இறைவனை அழைத்து துயிலெழுப்புகின்றது. இருவரின் நெறியும் வழியும் இறைநெறி ஆதலால் ஒருமைப்பாட்டு உணர்வுடன் அதனைப் பின்பற்றினால் வாழ்வில் உய்வு பெறலாம் என்பது உறுதியே.

\section{References}

Andal, (2010), Thiruppavai, Sri Vanni Vinayagar book stores, Madurai, India

Avvaiyar, Bharathiar, Bharadidasan, (2018), Aaathisoodi moolamum uraiyum, Saradha pathipagam, Chennai, India

Ilampuranar, (1982) Tholkappiyam porulathikaram, South India Saiva Siddhanta Works Publishing Society, Thirunelveli, India

Ilankumaranar, R., (2007) THirukural, Semamedu pthippagam, Colombo, Sri Lanka

Kamalakkannan, R.V., (2011) Naalayira Divya Prabandham, Moolamum thelivuraiyum, First edition, Varthamanan pathippagam, Chennai, India

\section{Funding: NIL}

Acknowledgement: NIL

Conflict of Interest: NIL

About the License:

\section{(c)(1)}

Attribution 4.0 International (CC BY 4.0)
(C) The author 2021. The text of this article is licensed under a Creative Commons Attribution 4.0 International License 